Gynecologic and

Obstetric Investigation
Gynecol Obstet Invest 2019;84:209-224

DOI: $10.1159 / 000495408$
Received: February 10, 2018

Accepted after revision: November 11, 2018

Published online: January 10, 2019

\title{
Nutrition as a Potential Factor of Primary Dysmenorrhea: A Systematic Review of Observational Studies
}

\author{
Zahra Bajalan $^{\text {a }}$ Zainab Alimoradi $^{a}$ Farnoosh Moafi ${ }^{b}$ \\ aSocial Determinants of Health Research Center, Qazvin University of Medical Sciences, Qazvin, Iran; \\ ${ }^{b}$ Department of Midwifery, School of Nursing and Midwifery, Qazvin University of Medical Sciences, Qazvin, Iran
}

\section{Keywords}

Nutrition · Primary dysmenorrhea - Systematic review .

Observational studies

\begin{abstract}
Background: The incidence and severity of primary dysmenorrhea are influenced by various factors. The aim of the present study was to review nutritional factors influencing primary dysmenorrhea. Methods: Academic databases including Web of Science, EMBASE, Scopus, and PubMed (including Medline) were searched using keywords of nutrition, diet, and primary dysmenorrhea. In this study, observational studies that were published in English from 1990 to April 2018, which focused on nutritional factors affecting primary dysmenorrhea, were selected. The evaluation of studies was performed using a modified STROBE checklist with 10 items. Results: Out of 5,814 retrieved studies, 38 articles met inclusion criteria and were included for final data synthesis. The increased consumption of fruits and vegetables as the sources of vitamins and minerals, as well as fish and milk and dairy products have positive associations with less menstrual pain. Inconsistent results were reported on the consumption
\end{abstract}

of other nutritional groups. Studies showed negative associations of meal skipping and following diet to lose weight with severity of dysmenorrhea. Conclusion: A few studies showed inconclusive findings due to methodological heterogeneities for assessing nutritional habits and different methods of measuring dysmenorrhea pain. Therefore, further analysis and future interventional studies with stronger methodologies are required.

(c) 2019 S. Karger AG, Basel

\section{Introduction}

Dysmenorrhea is the most common health-related problem $(60 \%)$ in women in the reproductive age $[1,2]$. It can reduce the quality of life and hinders social activities in young women, especially when it is accompanied by symptoms such as headache, fatigue, nausea and vomiting, diarrhea, boredom, chills and muscle cramps [3-5]. Primary dysmenorrhea is responsible for disability and inefficiency in terms of absence from the school or workplace. It has been reported that about $1 \%$ of women in the reproductive age lose their active work hours for 1-3 days

\section{KARGER}

(c) 2019 S. Karger AG, Basel

E-Mail karger@karger.com

www.karger.com/goi
Zainab Alimoradi

Department of Midwifery, School of Nursing and Midwifery

Qazvin University of Medical Science

Bahonar blv, Qazvin 34197-59811 (Iran)

E-Mail zainabalimoradi@qums.ac.ir 
per month. Also, about $14 \%$ of girls are absent from the school due to painful uterus contractions $[6,7]$. The feelings of shame and mood changes due to dysmenorrhea influence women's social relationships [8]. Also, the consequences and economic losses of medications may happen [8]. Therefore, dysmenorrhea is considered important in terms of its economic and social effect on women.

The increased production or unbalanced levels of prostaglandins creates pain due to increased uterine contractility, decreased uterine blood flow, and increased sensitivity of peripheral nerves $[2,7]$. Age, family history, body mass index, socioeconomic status, education, smoking, and alcohol use can influence the severity of primary dysmenorrhea [1,7-17]. Non-steroidal anti-inflammatory drugs and oral contraceptive pills have been listed as common treatments for women with dysmenorrhea [18]. The goal of treatment is to provide adequate pain relief and reduction of symptoms with least adverse effects. Marjoribanks et al. [19] in a systematic review reported that non-steroidal anti-inflammatory drugs were more effective than placebos, but some adverse effects including mild neurological (e.g., headache, drowsiness, dizziness) and gastrointestinal symptoms (e.g., nausea, indigestion) were increased. In another systematic review study, Proctor et al. [20] reported that COX-2 specific inhibitors effectively reduced dysmenorrhea, but they are not used in many countries due to cardiovascular effects.

Potential side effects of some drugs make some women with dysmenorrhea to seek alternative medicine such as herbal drugs, diet, fish oil supplements, vitamin E, and lowfat diets and vegetables to control their symptoms [21-23]. Two Cochran systematic reviews investigated the efficacy of dietary supplements including vitamin and herbal drugs on dysmenorrheal pain relief. They found that primary studies had low quality evidence to support the effectiveness of any dietary supplement for dysmenorrhea, and there was a lack of evidence of their safety $[24,25]$.

Besides the above-mentioned relative risk factors of dysmenorrhea, the potential role of women's daily diet in association with the severity of dysmenorrhea has been reported. Abdul-Razzak et al. [21] reported the positive effect of calcium on controlling primary dysmenorrhea. Di Cintio et al. [26] showed a poor positive relationship between egg and cheese consumption and dysmenorrhea. Balbi et al. [27] believed that an excessive consumption of fish, eggs, and fruits had a negative relationship with the reduction of dysmenorrhea. A study at Dumlupinar University reported that coffee consumption and family history of dysmenorrhea were some significant risk factors of dysmenorrhea [28]. In the study by Ozerdogan et al.
[29], the prevalence of dysmenorrhea was higher in students who consumed tea, Coca cola, or Pepsi. The severity of dysmenorrhea was higher in women with excessive sugar intake.

Given the probable relationship between the nutritional status (excluding supplements) and primary dysmenorrhea, as well as significance of knowledge of nutrition-related methods for the management of dysmenorrhea, this systematic review investigated the relationship between dietary habits and nutritional status with the intensity of primary dysmenorrhea.

\section{Methods}

\section{Search Process}

In accordance with the PRISMA guideline for designing and implementing systematic review studies [30], the following steps were taken to perform this study: systematic literature search, organization of documents for review, abstracting and quality assessment of documents, synthesizing data, and writing the final report.

\section{Search Strategy}

A systematic literature search was performed in databases including Web of Science, Embase, Scopus, and PubMed (including Medline). The Boolean search method was used using keywords that were connected together via AND, OR, and NOT: ([Risk factor OR Associate factor OR contributing factor] OR [Food OR nutrient OR Diet OR Nutrition Status OR Nutrition Assessments OR Nutrition OR Nutrition Indexes OR Nutritional Indices OR Prognostic Nutritional Index OR Mini Nutritional Assessment]) AND (Dysmenorrhea OR Menstruating Disturbances OR Menstrual Disorders OR Menstrual Disorder OR Pelvic Pain OR Painful Menstruation OR Painful Period OR Period Pain OR Primary Dysmenorrhea OR Menstrual Pain OR Menstrual Cramps). Also, the reference list of included studies and previous systematic reviews were searched to increase the search coverage.

\section{Inclusion Criteria}

Type of Studies

Observational studies including cross-sectional, case-control, and cohort studies published in English language from 1990 to April 2018 were included. The studies published before 1990 and in languages other than English were excluded. Moreover, case reports, letters to the editor, and review articles were excluded. Trial studies on dietary supplements and herbal drugs were excluded because of the presence of a recent published systematic review on this topic [24].

\section{Subjects}

They were from all age ranges which were suffering from primary dysmenorrhea in their menstrual cycles. Those subjects with secondary dysmenorrhea who used contraceptive methods such as intrauterine contraceptive device or oral contraceptive pills were excluded. If the type of dysmenorrhea was not specified (due to a higher prevalence of primary dysmenorrhea in comparison to secondary dysmenorrhea), data was included. 


\section{Exposure}

Observational studies on nutritional habits, daily food consumption, or nutrients in the daily diet were included. Trial studies on dietary supplements and herbal drugs were excluded.

\section{Outcomes Measure}

Intensity of dysmenorrheal pain was assessed using a visual analogue scale. Also, other valid scales or dichotomous measurements were other main outcomes measured in this systematic review.

\section{Study Selection}

The title and abstract of all articles retrieved during the search process were studied using the inclusion criteria. The full texts of the articles were examined using the above-mentioned criteria independently by 2 authors. Disagreements were resolved through discussions.

\section{Quality Assessment}

The modified STROBE checklist was used to assess the quality of selected observational studies with cohort, case-control, and cross-sectional designs. The STROBE as a valid instrument with 22 items was used for reporting or evaluating observational studies [31]. For the quality assessment of this review, the modified STROBE checklist with 10 items was used in relation of the methodological assessment of studies. The modified checklist consisted of objectives (specific objectives), study design (key elements of the study design), setting (the setting, location, relevant date, period of recruitment, exposure, follow-up, and data collection), subjects (eligibility criteria and the sources and methods of samples' selection), definition and measurement of all variables (outcomes, exposures, predictors for each variable of interest, sources of data, and methods of measurement), potential sources of bias and confounders (efforts taken to address potential sources of bias), sample size (final sample size), quantitative variables (handling variables during analyses, group selections, and reasons), and statistical methods (methods used to control confounding variables, methods used to examine subgroups and interactions, addressing missing data). Next, the studies were scored as "low risk", if the above mentioned criteria were unmet they were scored as "high risk", and if these items were not explained at all, they were rated as "not clearly defined". The quality assessment process was performed independently by 2 authors and disagreements were resolved through discussions.

\section{Data Extraction}

After screening and examining the quality of selected studies, data was extracted and recorded in forms independently by 2 authors. The forms were consisted of questions about the name of the first author, year, study design, country of the study, sample size, age of subjects, associations between dysmenorrhea and nutritional groups, and nutritional habits.

\section{Data Synthesis}

The qualitative synthesis of findings was performed due to the heterogeneity of measures used to assess and report nutrition consumption and insufficiency in provided data. Also, different measures were used to report the intensity of dysmenorrhea.

Nutrition as a Potential Factor of Primary

Dysmenorrhea

\section{Findings}

The search process led to retrieving 5,814 potentially relevant articles. Of which, 157 articles were found related to the study topic during abstract and title checking, but 18 articles were duplicated and 3 articles were published in other languages than English. The full texts of 136 remaining articles were reviewed and 38 articles were selected based on the inclusion criteria. The reference list of the included studies was read to find potentially related studies, but no article was included in the search process. Figure 1 showed the search process according to the PRISMA flowchart.

\section{Description of Selected Studies}

Most articles $(n=27)$ had cross-sectional designs, 4 had prospective or cohort designs, and the remaining were case-control studies. A total of 19,626 women were studied in these studies. The largest number of samples was 2,166 individuals in the study by Fujiwara and Nakata [32] in Japan. Also, the study of Abdul-Razzak et al. [33] in Jordan had the lowest number of samples $(n=56)$. The studies were performed in the different parts of the world including the United States, China, Japan, Iran, Jordan, Nepal, Turkey, India, South Korea, Ethiopia, Georgia, Serbia, and Italy. Japan had the largest number of studies with 6 articles (Table 1).

\section{Quality Assessment}

Evaluation of the selected studies using the modified checklist of STORBE with 10 items showed that the studies mostly had a moderate quality level. The quality assessment scores were presented in Table 1. The majority of the studies had issues in reporting bias, sample size estimation, description of design, and statisti$\mathrm{cal}$ analysis of quantitative variables. Figure 2 showed the graphical representation of the quality of studies.

\section{Outcome Measurement}

The findings of the selected studies were presented in 2 parts: (i) summarizing the results of the studies regarding the association between dysmenorrhea and dietary nutritional groups based on the food pyramid (Table 2) and (ii) abstracting the results of the studies based on nutritional and dietary habits (Table 3).

\section{The Results of the Selected Studies According to the Dietary}

Nutritional Group

Carbohydrate Including Bread and Cereals: Consumption of pasta, rice, white bread, biscuits, pastry, potatoes, grains were studied by Di Cintio et al. [26], Balbi et al. [27], Tavallaee et al. [16], and Gagua et al. [6], but no association was reported between the consumption of food related to these groups and dysmenorrhea.

Fruits and Vegetables: The association between the consumption of fruits and vegetables were investigated in 4 studies, but Di Cintio et al. [26] and Abu Helwa et al. [55] found no associations. However, Tavallaee et al. [16] and Balbi et al. [27] reported that fruit consumption had negative associations with the level of menstrual pain.

Meat, Legumes, Eggs, and Nuts: This group consisted of a wide range of nutrients including meat, poultry, fish, legumes such as beans and soybean, eggs, and nuts. Fish consumption as a separate nutrient was studied by Deutch [34], Balbi et al. [27], and Grandi et al. [44] which reported inconsistent results. Deutch [34] found that a high intake of $n-3$ fatty acids through marine sources was 


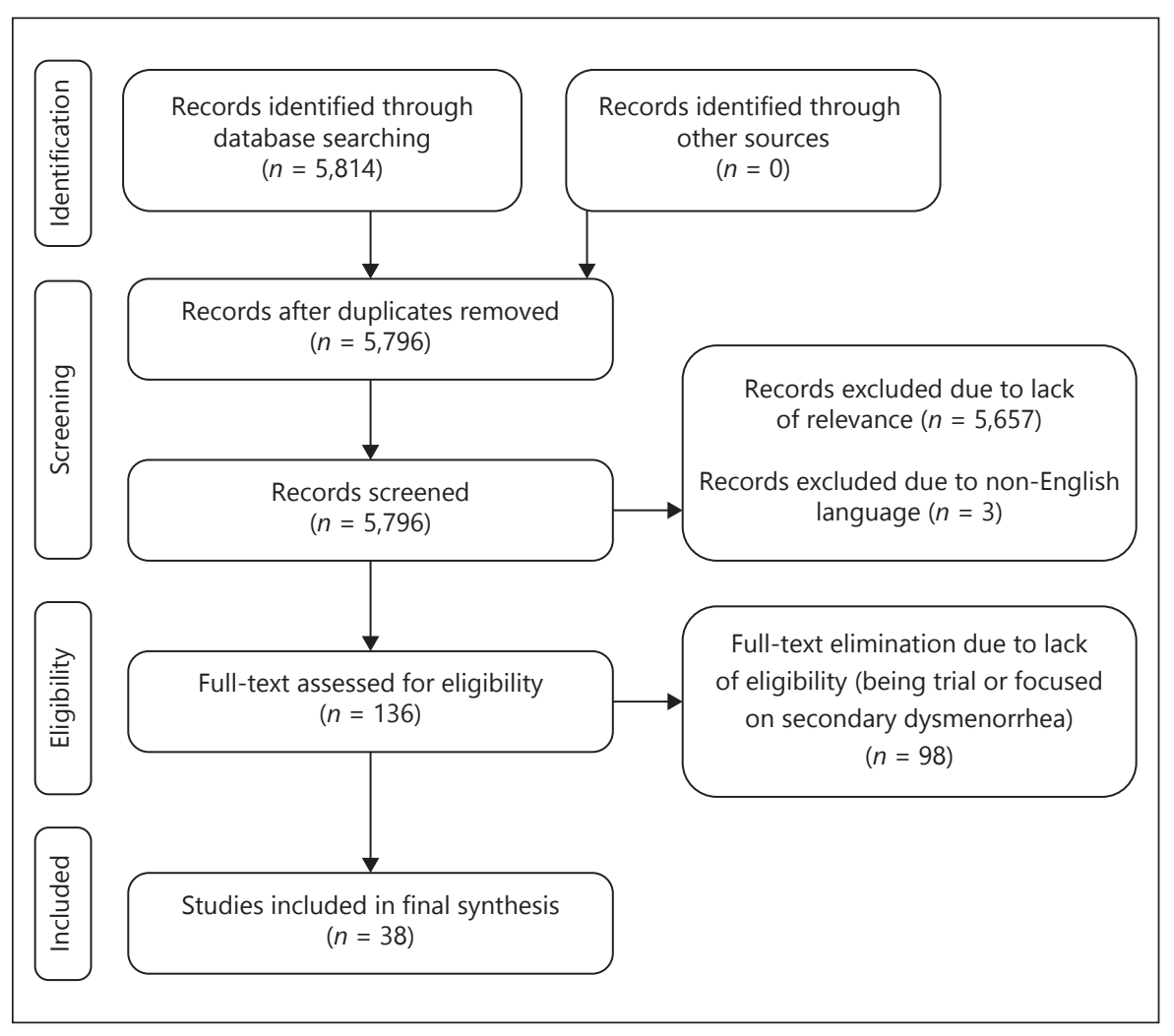

Fig. 1. PRISMA flow diagram.

reported that women with a low fat diet significantly experienced

associated with pain reduction; also Balbi et al. [27] found that women with dysmenorrhea significantly consumed less fish compared to healthy controls, but Grandi et al. [44] did not find any significant association. Egg as another nutrient was studied by $\mathrm{Di}$ Cintio et al. [26] and Balbi et al. [27] with completely contradicting results. Di Cintio et al. [26] reported that women with dysmenorrhea consumed eggs more frequently than healthy controls, but Balbi et al. [27] reported that women with dysmenorrhea consumed significantly less eggs compared to healthy controls. Other studies did not find significant results concerning the association between dysmenorrhea and the consumption of meat and its alternatives [16, 26, 27, 39, 52, 55].

Milk and Dairy: Milk and dairy consumption were reported in several studies. Four studies found a significant inverse association between the consumption of dairy products and dysmenorrhea [16, 21, 33, 59], Di Cintio et al. [26] found no relationship between the intake of milk and risk of dysmenorrhea, but cheese was more frequently used by women with dysmenorrhea than healthy controls.

Other Products: Other products included sugar, fat, salt, caffeine, vitamins, and minerals:

A) Sugar: Consumption of sugar in 3 studies had a significant relationship with dysmenorrhea. It developed a significantly higher risk of dysmenorrhea in women consuming excessive amount of sugar $[6,29,60]$. Inconsistently, Tavallaee et al. [16], Grandi et al. [44], Mohamadirizi and Kordi [52], Abu Helwa et al. [55] found no association between the intake of sugar and dysmenorrhea.

B) Fats: Fat consumption was reported by 2 studies. Nagata et al. [39] found that only the intake of saturated fat had a positive association with lower menstrual pain. Also, Tavallaee et al. [16] less pain during menstrual periods.

C) Salt: Three studies examined the relationship between excessive salt intake and dysmenorrhea. One study reported a higher prevalence of dysmenorrhea in women with moderate or excessive intake of salt compared with those with a minimal intake [29]. Two other studies did not report a significant association between a high salt intake and dysmenorrhea $[44,55]$.

D) Caffeine Consumption: Caffeine consumption was the most studied nutrient including coffee, tea, Nescafe, carbonated soft drinks, chocolate, and hot chocolate. In 15 studies, the association between caffeine intake and dysmenorrhea was assessed. In some studies, some forms of caffeinated drinks were assessed separately such as tea, coffee, Nescafe, but others studies assessed this group as whole. Seven studies [16, 29, 38, 44, 48, 52, 55] did not find any significant association between caffeine intake and dysmenorrhea, but Faramarzi and Salmalian [49], Pejčić and Jankovic [57], and Hailemeskel et al. [56] found significant associations between caffeine consumption and higher intensity of menstrual pain. Drinking tea did not have a significant association with dysmenorrhea in 4 studies [28, 43, 45, 60], but Wang et al. [37] reported more tea consumption among women with dysmenorrhea. The association between coffee and menstrual pain was reported in 3 studies $[28,43,60]$ indicating a positive association, but 7 studies [45] reported that coffee consumption had no association with dysmenorrhea.

Vitamins and Minerals: Venkata et al. [42] reported reduced blood levels of Vitamin E and C and primary dysmenorrhea. Consistently, Pramanik et al. [53] reported a higher daily intake of beta carotene, vitamin-E, and zinc in girls without dysmenorrhea than 
Table 1. Characteristics of selected primary studies

\begin{tabular}{|c|c|c|c|c|c|c|c|c|}
\hline No. & Author, year & Study Design & Country & $\begin{array}{l}\text { No. of } \\
\text { participants }\end{array}$ & $\begin{array}{l}\text { Age } \\
\text { group, years }\end{array}$ & $\begin{array}{l}\text { STROBE } \\
\text { score }\end{array}$ & $\begin{array}{l}\text { Outcome } \\
\text { measured }\end{array}$ & Nutritional assessment \\
\hline 1 & $\begin{array}{l}\text { Deutch [34], } \\
1995\end{array}$ & Cross sectional & Denmark & 181 & $20-45$ & 6 & $\begin{array}{l}\text { Menstrual pain: } \\
\text { have/not have }\end{array}$ & $\begin{array}{l}\text { Two (prospective) 4-day } \\
\text { dietary records were used to } \\
\text { estimate average daily } \\
\text { nutrient intake }\end{array}$ \\
\hline 2 & $\begin{array}{l}\text { Montero [35], } \\
1996\end{array}$ & Cross sectional & Spain & 1147 & $14-20$ & 8 & $\begin{array}{l}\text { Self-report of perceived } \\
\text { pain as severe/medium/ } \\
\text { mild/no pain }\end{array}$ & $\begin{array}{l}\text { Asked whether they had } \\
\text { tried to lose weight }\end{array}$ \\
\hline 3 & $\begin{array}{l}\text { Di Cintio [26], } \\
1997\end{array}$ & Case control & Italy & 251 & $\begin{array}{l}\text { Median: } \\
26-27\end{array}$ & 8.5 & $\begin{array}{l}\text { Andersch and Milsom's } \\
\text { classification on } \\
\text { dysmenorrhea }\end{array}$ & $\begin{array}{l}\text { Weekly frequencies of intake } \\
\text { of each food item without } \\
\text { information on portion size }\end{array}$ \\
\hline 4 & $\begin{array}{l}\text { Balbi et al. [27], } \\
2000\end{array}$ & Cross sectional & Naples & 356 & $14-21$ & 8 & $\begin{array}{l}\text { Intensity of the pain } \\
\text { assessed with VAS }\end{array}$ & $\begin{array}{l}\text { Frequency which certain } \\
\text { foodstuffs were consumed }\end{array}$ \\
\hline 5 & $\begin{array}{l}\text { Fujiwara [36], } \\
2003\end{array}$ & Case control & Japan & 439 & $18-20$ & 7.5 & $\begin{array}{l}\text { Intensity of dysmenorrhea } \\
\text { classified into score 1: } \\
\text { free of pain or painful, but } \\
\text { without need for analgesic; } \\
\text { score 2: painful, } \\
\text { requiring analgesic; score } 3 \text { : } \\
\text { painful, not relieved by } \\
\text { analgesic }\end{array}$ & $\begin{array}{l}\text { Grouped as having breakfast } \\
\text { every morning, having } \\
\text { breakfast } 1-6 \text { times per week, } \\
\text { having breakfast less than } \\
\text { once a week) }\end{array}$ \\
\hline 6 & $\begin{array}{l}\text { Wang et al. [37], } \\
2004\end{array}$ & Cohort & China & 388 & $20-34$ & 10 & Menstrual pain (yes or no) & $\begin{array}{l}\text { Tea consumption but not } \\
\text { mentioned how measure }\end{array}$ \\
\hline 7 & $\begin{array}{l}\text { Weissman } \\
\text { et al. [17], } 2004\end{array}$ & Cohort & USA & 404 & $19-46$ & 8 & $\begin{array}{l}\text { Four-point scale: no pain, } \\
\text { minimal (can work } \\
\text { somewhat uncomfortable), } \\
\text { moderate (can work, but } \\
\text { quite uncomfortable), or } \\
\text { severe (miss work, have } \\
\text { to be in bed) }\end{array}$ & $\begin{array}{l}\text { Ask about vitamins use to } \\
\text { cope with dysmenorrhea }\end{array}$ \\
\hline 8 & $\begin{array}{l}\text { Chung } \\
\text { et al. [38], } \\
2005\end{array}$ & Cross sectional & Taiwan & 151 & $21-44$ & 7 & $\begin{array}{l}\text { Experience of } \\
\text { dysmenorrhea as no/ } \\
\text { seldom/always }\end{array}$ & $\begin{array}{l}\text { Drinking coffee habit as } \\
\text { yes/no/quit }\end{array}$ \\
\hline 9 & $\begin{array}{l}\text { Nagata } \\
\text { et al. [39], } 2005\end{array}$ & Cross sectional & Japan & 276 & $19-24$ & 8 & $\begin{array}{l}\text { Severity of menstrual pain } \\
\text { using Andersch and Milson } \\
\text { scoring system }\end{array}$ & $\begin{array}{l}\text { Intakes of soy, fat } \\
\text { and dietary fiber by } \\
\text { assessing average } \\
\text { frequency consumption } \\
\text { of } 169 \text { food } \\
\text { during the year }\end{array}$ \\
\hline 10 & $\begin{array}{l}\text { Fujiwara } \\
\text { et al. [40], } 2007\end{array}$ & Cross sectional & Japan & 716 & $18-20$ & 6.5 & $\begin{array}{l}\text { Intensity of dysmenorrhea } \\
\text { classified into score 1: } \\
\text { free of pain or painful, but } \\
\text { without need for analgesic; } \\
\text { score 2: painful, } \\
\text { requiring analgesic; score 3: } \\
\text { painful, not relieved } \\
\text { by analgesic }\end{array}$ & $\begin{array}{l}\text { Dietary habits were } \\
\text { classified into having no } \\
\text { experience with dieting/ } \\
\text { currently on a diet/with } \\
\text { a history of dieting }\end{array}$ \\
\hline 11 & $\begin{array}{l}\text { Ozerdogan } \\
\text { et al. [29], } 2009\end{array}$ & Cross sectional & Turkey & 857 & $17-32$ & 8 & $\begin{array}{l}\text { Severity of menstrual } \\
\text { pain using } \\
\text { Andersch } \\
\text { and Milson scoring system }\end{array}$ & $\begin{array}{l}\text { Sugar and salt consumption } \\
\text { categorized into minimal, } \\
\text { moderate, or excessive } \\
\text { daily intake. And caffeine } \\
\text { consumption as } \\
\text { infrequently/frequently }\end{array}$ \\
\hline
\end{tabular}


Table 1 (continued)

\begin{tabular}{|c|c|c|c|c|c|c|c|c|}
\hline No. & Author, year & Study Design & Country & $\begin{array}{l}\text { No. of } \\
\text { participants }\end{array}$ & $\begin{array}{l}\text { Age } \\
\text { group, years }\end{array}$ & $\begin{array}{l}\text { STROBE } \\
\text { score }\end{array}$ & $\begin{array}{l}\text { Outcome } \\
\text { measured }\end{array}$ & Nutritional assessment \\
\hline 12 & $\begin{array}{l}\text { Fujiwara } \\
\text { et al. [40], } 2009\end{array}$ & Cross sectional & Japan & 315 & $18-20$ & 7 & $\begin{array}{l}\text { Intensity of dysmenorrhea } \\
\text { classified into score } 1 \text { : free of } \\
\text { pain or painful, but without } \\
\text { need for analgesic; score } 2 \text { : } \\
\text { painful, requiring analgesic; } \\
\text { score 3: painful, not relieved } \\
\text { by analgesic }\end{array}$ & $\begin{array}{l}\text { Categorized in } 6 \text { groups: I/ } \\
\text { eating breakfast; II/skipping } \\
\text { breakfast; III/not eating fast } \\
\text { foods; IV/eating fast foods; } \\
\text { V/not eating processed foods; } \\
\text { VI/eating processed foods }\end{array}$ \\
\hline 13 & $\begin{array}{l}\text { Fujiwara and } \\
\text { Nakata [41], } \\
2010\end{array}$ & Prospective & Japan & 945 & $18-20$ & 7.5 & $\begin{array}{l}\text { Intensity of dysmenorrhea } \\
\text { classified into score } 1 \text { : free of } \\
\text { pain or painful, but without } \\
\text { need for analgesic; score } 2 \text { : } \\
\text { painful, requiring analgesic; } \\
\text { score 3: painful, not relieved } \\
\text { by analgesic }\end{array}$ & $\begin{array}{l}\text { Group I: } \\
\text { Having breakfast every } \\
\text { morning; Group II: having } \\
\text { breakfast } 1-6 \\
\text { times a week; and group III, } \\
\text { having breakfast less than } \\
\text { once a week }\end{array}$ \\
\hline 14 & $\begin{array}{l}\text { Abdul-Razzak } \\
\text { et al. [21], } \\
2010\end{array}$ & Cross sectional & Jordan & 127 & $19-24$ & 7.5 & $\begin{array}{l}\text { Pain severity as mild: pain } \\
\text { that resolved without the } \\
\text { need for medication. } \\
\text { Severe: pain that is resolved } \\
\text { with simple analgesics } \\
\text { Very severe: pain that is not } \\
\text { relieved with simple } \\
\text { analgesics and may interfere } \\
\text { with usual daily activities }\end{array}$ & $\begin{array}{l}\text { Frequency and type of diary } \\
\text { product intake (milk, yogurt, } \\
\text { cheese, and labanah) } \\
\text { recorded based on daily } \\
\text { intake of dairy products }\end{array}$ \\
\hline 15 & $\begin{array}{l}\text { Unsal } \\
\text { et al. [28], } \\
2010\end{array}$ & Cross sectional & Turkey & 623 & $17-30$ & 8 & $\begin{array}{l}\text { Severity of dysmenorrhea } \\
\text { assessed by VAS and } \\
\text { Andersch and Milson } \\
\text { scoring system }\end{array}$ & $\begin{array}{l}\text { Tea consuming: at least } 4 \\
\text { glasses of tea in a day } \\
\text { ( } 75 \mathrm{~mL}) \text {, coffee consuming: } \\
\text { at least } 3 \text { cups of coffee in a } \\
\text { day ( } 150 \mathrm{~mL}) \text {, cola } \\
\text { consuming: at least a glass } \\
\text { of cola in a day ( } 200 \mathrm{~mL}) \text {, } \\
\text { chocolate eating: at least } 2 \\
\text { bars of chocolate in a day } \\
(150 \mathrm{~mL})\end{array}$ \\
\hline 16 & $\begin{array}{l}\text { Venkata } \\
\text { et al. [42], } 2011\end{array}$ & Case control & China & 191 & $18-23$ & 4.5 & $\begin{array}{l}\text { Having dysmenorrhea: } \\
\text { yes/no }\end{array}$ & $\begin{array}{l}\text { Plasma vitamin } \mathrm{C} \text {, plasma } \\
\text { vitamin } \mathrm{E}\end{array}$ \\
\hline 17 & $\begin{array}{l}\text { Bin Mahmoud } \\
\text { et al. [43], } \\
2014\end{array}$ & Cross sectional & $\begin{array}{l}\text { Saudi } \\
\text { Arabia }\end{array}$ & 384 & $17-45$ & 9.5 & $\begin{array}{l}\text { Pain as one of menstrual } \\
\text { symptoms }\end{array}$ & $\begin{array}{l}\text { Asked about usual daily } \\
\text { consumption of the } \\
\text { caffeinated beverages coffee, } \\
\text { tea, Nescafe, carbonated soft } \\
\text { drinks, chocolate and hot } \\
\text { chocolate, in number of } \\
\text { cups or cans per day }\end{array}$ \\
\hline 18 & $\begin{array}{l}\text { Tavallaee } \\
\text { et al. [16], } \\
2011\end{array}$ & Cross sectional & Iran & 381 & $16-56$ & 7.5 & $\begin{array}{l}\text { Severity of dysmenorrhea } \\
\text { assessed by Anderch and } \\
\text { Milson scoring system }\end{array}$ & $\begin{array}{l}\text { Consumption of fruits and } \\
\text { vegetables/milk products/ } \\
\text { meat and alternatives/ } \\
\text { grains/fats and oils/ } \\
\text { sweets and chocolates/ } \\
\text { caffeine but not mentioned } \\
\text { how measured }\end{array}$ \\
\hline 19 & $\begin{array}{l}\text { Gagua } \\
\text { et al. [6], } 2012\end{array}$ & Cross sectional & Georgia & 431 & $14-20$ & 7 & $\begin{array}{l}\text { Having dysmenorrhea: } \\
\text { yes/no }\end{array}$ & $\begin{array}{l}\text { Asked about meal } \\
\text { skipping: yes/no }\end{array}$ \\
\hline 20 & $\begin{array}{l}\text { Grandi } \\
\text { et al. [44], } \\
2012\end{array}$ & Cross sectional & Italy & 408 & Mean 22.9 & 9.5 & Pain intensity using a VAS & $\begin{array}{l}\text { Sugar and salt consumption } \\
\text { (minimal, moderate, } \\
\text { excessive) and breakfast } \\
\text { frequency (daily, } 2 / 3 \text { times } \\
\text { a week, never) }\end{array}$ \\
\hline 21 & $\begin{array}{l}\text { Seven et al. [45], } \\
2013\end{array}$ & Case control & Turkey & 380 & $18-23$ & 7.5 & Pain intensity using a VAS & $\begin{array}{l}\text { Eating chocolate: } \\
\text { yes / no }\end{array}$ \\
\hline
\end{tabular}


Table 1 (continued)

\begin{tabular}{|c|c|c|c|c|c|c|c|c|}
\hline No. & Author, year & Study Design & Country & $\begin{array}{l}\text { No. of } \\
\text { participants }\end{array}$ & $\begin{array}{l}\text { Age } \\
\text { group, years }\end{array}$ & $\begin{array}{l}\text { STROBE } \\
\text { score }\end{array}$ & $\begin{array}{l}\text { Outcome } \\
\text { measured }\end{array}$ & Nutritional assessment \\
\hline 22 & $\begin{array}{l}\text { Fujiwara and } \\
\text { Nakata [32], } \\
2013\end{array}$ & Prospective & Japan & 2,166 & $18-20$ & 5.5 & $\begin{array}{l}\text { Intensity of dysmenorrhea } \\
\text { classified into score 1: free } \\
\text { of pain or painful, but } \\
\text { without need for analgesic; } \\
\text { score 2: painful, requiring } \\
\text { analgesic; score 3: painful, } \\
\text { not relieved by analgesic }\end{array}$ & Skipping breakfast: yes/no \\
\hline 23 & $\begin{array}{l}\text { Vani et al. [46], } \\
2013\end{array}$ & Cross sectional & India & 853 & $13-19$ & 8 & $\begin{array}{l}\text { Occurrence of } \\
\text { dysmenorrhea and if } \\
\text { present, is it severe enough } \\
\text { to skip classes or any need } \\
\text { to take medications }\end{array}$ & $\begin{array}{l}\text { Junk food consumption was } \\
\text { assessed according frequency } \\
\text { of eating junk per week and } \\
\text { Fussy behavior at eating food } \\
\text { and eating less food in order } \\
\text { to lose weight }\end{array}$ \\
\hline 24 & $\begin{array}{l}\text { Gangwar et al. } \\
{[47], 2014}\end{array}$ & Cross sectional & India & 101 & $18-25$ & 6 & Pain intensity using a VAS & $\begin{array}{l}\text { Diet as vegetarian/non- } \\
\text { vegetarian }\end{array}$ \\
\hline 26 & $\begin{array}{l}\text { Abdul-Razzak } \\
\text { et al. [33], } 2014\end{array}$ & Cross sectional & Jordan & 56 & $17-24$ & 6 & $\begin{array}{l}\text { Pain intensity using a } \\
\text { VAS and graded as mild/ } \\
\text { moderate/ sever/very sever }\end{array}$ & $\begin{array}{l}\text { Frequency and type of dairy } \\
\text { product intake (milk, yogurt, } \\
\text { cheese, and labanah) } \\
\text { recorded on daily basis }\end{array}$ \\
\hline 27 & $\begin{array}{l}\text { Faramarzi and } \\
\text { Salmalian [49], } \\
2014\end{array}$ & Cross sectional & Iran & 360 & $17-25$ & 8 & $\begin{array}{l}\text { Graded as } 0=\text { absence of } \\
\text { dysmenorrhea. } \\
1=\text { mild pain, } \\
2=\text { moderate pain, } \\
3=\text { severe pain }\end{array}$ & $\begin{array}{l}\text { Caffeine intake defined as } \\
\text { excessive if consumption of } \\
\text { caffeinated soft drinks, coffee, } \\
\text { decaffeinated coffee, tea, } \\
\text { chocolate milk, and chocolate } \\
\text { bars in the daily diet was } \\
\text { self-reported as } \geq 300 \mathrm{mg} / \text { day } \\
\text { and otherwise it was as } \\
\text { minimum to moderate. } \\
\text { Having breakfast was } \\
\text { classified as normal if } \\
\text { participant had breakfast one } \\
\text { to six times per week and as } \\
\text { low if she had breakfast less } \\
\text { than once a week }\end{array}$ \\
\hline 29 & $\begin{array}{l}\text { Kazama } \\
\text { et al. [51], } 2015\end{array}$ & Cross sectional & Japan & 1,018 & $12-15$ & 6.5 & $\begin{array}{l}\text { Pain intensity using } \\
\text { a VAS }\end{array}$ & $\begin{array}{l}\text { Habitual } \\
\text { breakfast, lunch, and supper } \\
\text { classified as (1) everyday, ( } 2 \text { ) } \\
\text { sometimes, } \\
\text { and (3) none }\end{array}$ \\
\hline 30 & $\begin{array}{l}\text { Mohamadirizi } \\
\text { and Kordi [52], } \\
2015\end{array}$ & Cross sectional & Iran & 407 & $10-15$ & 9 & $\begin{array}{l}\text { Pain as one dimension } \\
\text { of menstrual distress } \\
\text { questionnaire }\end{array}$ & $\begin{array}{l}\text { The nutrition pattern } \\
\text { questionnaire included } 4 \text { main } \\
\text { nutrients: sweet-fatty } \\
\text { (chocolates, cake, ice cream, } \\
\text { nuts, etc.), salty-fatty (soya, } \\
\text { red meat, chicken meat, etc.), } \\
\text { fast foods (hamburger, pizza, } \\
\text { potato chips, etc.), and } \\
\text { caffeine-containing materials } \\
\text { (tea, coffee, etc.). The } \\
\text { frequency of their } \\
\text { consumption within the past } \\
\text { year was investigated }\end{array}$ \\
\hline
\end{tabular}


Table 1 (continued)

\begin{tabular}{|c|c|c|c|c|c|c|c|c|}
\hline No. & Author, year & Study Design & Country & $\begin{array}{l}\text { No. of } \\
\text { participants }\end{array}$ & $\begin{array}{l}\text { Age } \\
\text { group, years }\end{array}$ & $\begin{array}{l}\text { STROBE } \\
\text { score }\end{array}$ & $\begin{array}{l}\text { Outcome } \\
\text { measured }\end{array}$ & Nutritional assessment \\
\hline 31 & $\begin{array}{l}\text { Pramanik } \\
\text { et al. [53], } 2015\end{array}$ & Case control & India & 379 & $12-17$ & 6.5 & $\begin{array}{l}\text { Having dysmenorrhea: } \\
\text { yes/no }\end{array}$ & $\begin{array}{l}24 \mathrm{~h} \text { food consumption } \\
\text { recall }\end{array}$ \\
\hline 32 & $\begin{array}{l}\text { Bavil et al. [54], } \\
2016\end{array}$ & Cross sectional & Iran & 250 & $18-26$ & 7.5 & $\begin{array}{l}\text { Having dysmenorrhea: } \\
\text { yes/no }\end{array}$ & $\begin{array}{l}\text { Eating behavior but not } \\
\text { clearly explained how } \\
\text { measured }\end{array}$ \\
\hline 33 & $\begin{array}{l}\text { Abu Helwa } \\
\text { et al. [55], } 2018\end{array}$ & Cross sectional & Palestine & 956 & Mean 19.7 & 8.5 & Pain intensity using a VAS & $\begin{array}{l}\text { Nutritional habits as having } \\
\text { breakfast every day/ } \\
\text { sometimes/never and habits } \\
\text { about consumption of tea/ } \\
\text { coffee/nescafe ( } \leq 1 \text { cup or } \\
\geq 2 \text { cup)/ consumption of } \\
\text { Vegetables and fruits, meat } \\
\text { and protein rich diet, sweets } \\
\text { and sugary beverages, salty } \\
\text { foods graded as never or low/ } \\
\text { moderate/high/very high } \\
\text { over last } 6 \text { months }\end{array}$ \\
\hline 34 & $\begin{array}{l}\text { Hailemeskel } \\
\text { et al. [56], } 2016\end{array}$ & Cross sectional & Ethiopia & 440 & Mean 20.6 & 7.5 & $\begin{array}{l}\text { Severity of dysmenorrhea } \\
\text { assessed by VAS and } \\
\text { Anderch and Milson } \\
\text { scoring system }\end{array}$ & $\begin{array}{l}\text { Ask about history of attempt } \\
\text { to lose weight: yes/no }\end{array}$ \\
\hline 35 & $\begin{array}{l}\text { Pejčić and } \\
\text { Jankovic [57], } \\
2016\end{array}$ & Case control & Serbia & 288 & $18-29$ & 9.5 & Dysmenorrhea yes/no & $\begin{array}{l}\text { Ask about Consuming at } \\
\text { least one cup of coffee daily }\end{array}$ \\
\hline 36 & $\begin{array}{l}\text { Shinde and } \\
\text { Laddad [58], } \\
2016\end{array}$ & Cross sectional & India & 624 & $11-19$ & 6 & Dysmenorrhea yes/no & $\begin{array}{l}\text { Frequency of eating junk food } \\
\text { (categorized into }<3 \text { days per } \\
\text { week and }>3 \text { days per week }\end{array}$ \\
\hline 37 & $\begin{array}{l}\text { Karacin } \\
\text { et al. [59], } \\
2018\end{array}$ & Case control & Turkey & 683 & $18-25$ & 9 & $\begin{array}{l}\text { Severity of dysmenorrhea } \\
\text { assessed by VAS }\end{array}$ & $\begin{array}{l}\text { The intake of dairy products } \\
\text { assessed on daily basis }\end{array}$ \\
\hline 38 & $\begin{array}{l}\text { Muluneh } \\
\text { et al. [60], } \\
2018\end{array}$ & Cross sectional & Ethiopia & 539 & $14-24$ & 9.5 & Dysmenorrhea yes/no & $\begin{array}{l}\text { Sugar intake: Excessive if } \\
\text { individuals took } 12 \text { or more } \\
\text { teaspoons of table sugar daily, } \\
\text { Moderate if } 6 \text { to } 12 \text { teaspoons; } \\
\text { and in a restricted use if less } \\
\text { than } 6 \text { teaspoons }\end{array}$ \\
\hline
\end{tabular}

Fig. 2. Quality assessment of selected studies according to STROBE checklist.

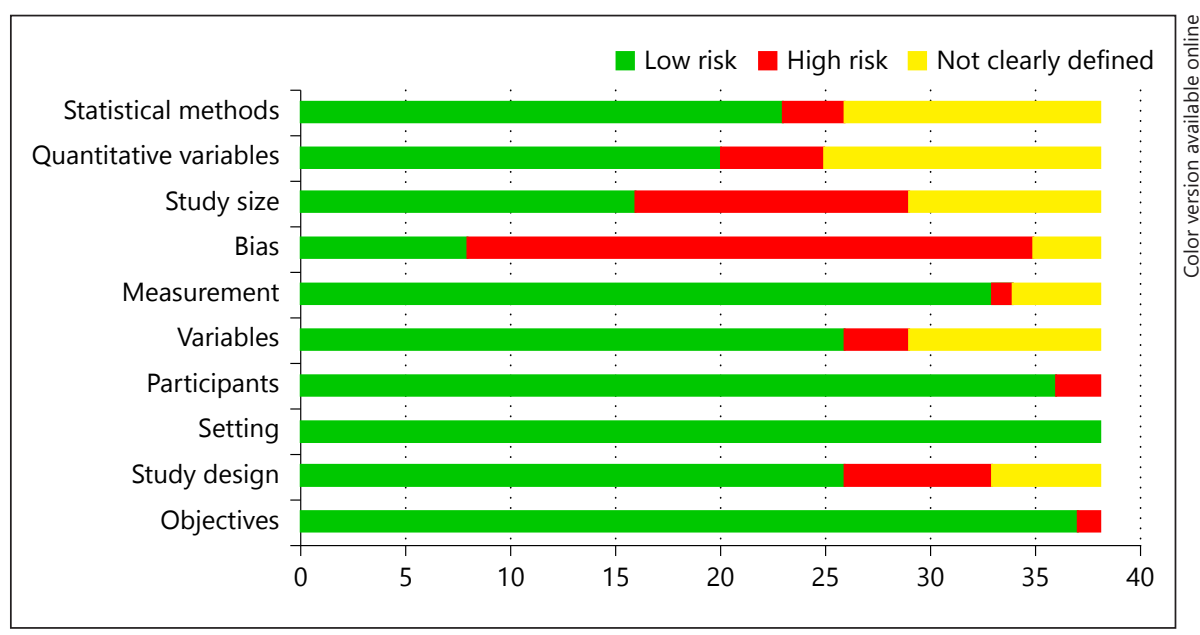


Table 2. Summarized results of reviewed studies according to dietary nutritional group and habit

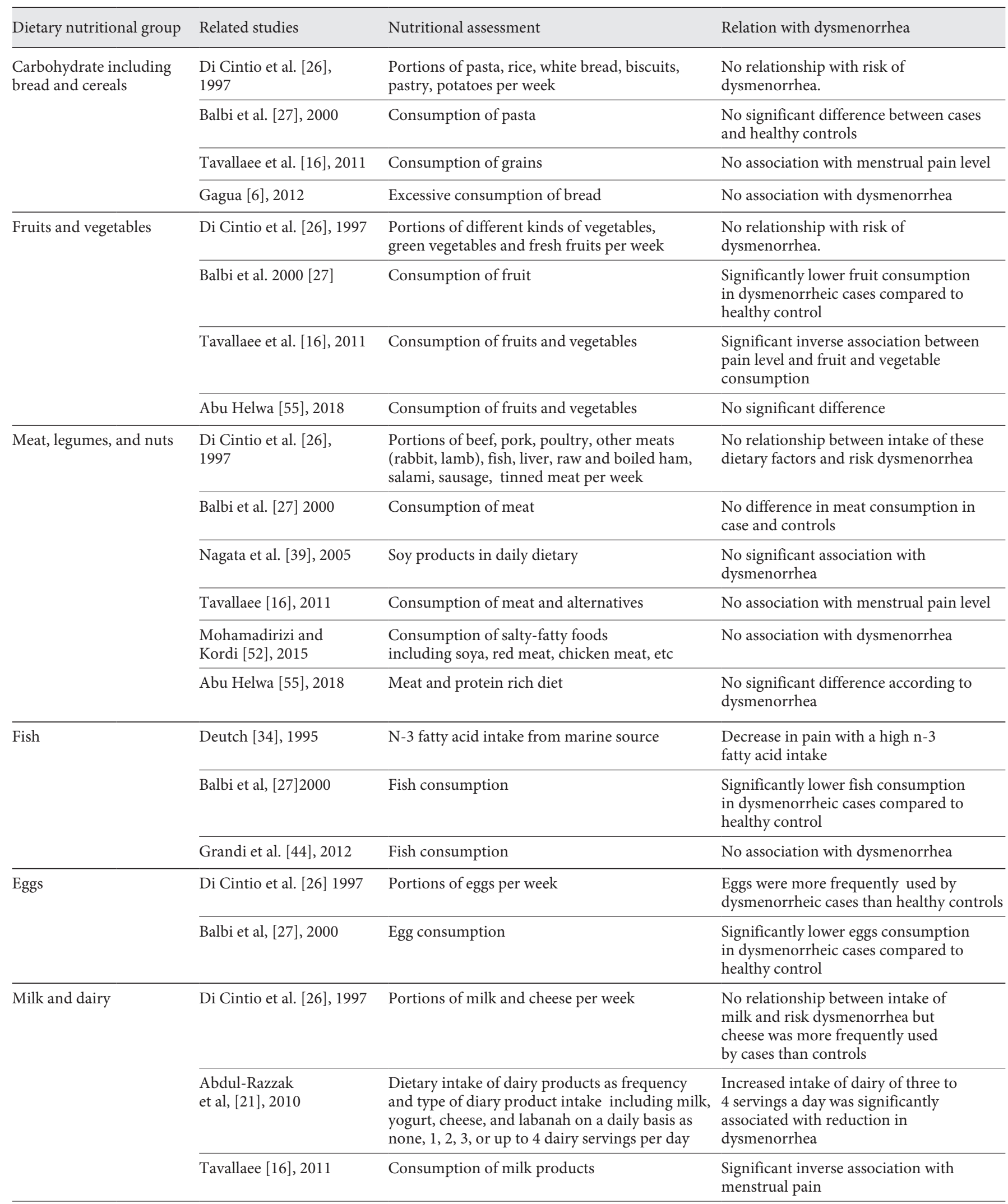


Table 2 (continued)

\begin{tabular}{|c|c|c|c|c|}
\hline \multicolumn{2}{|c|}{ Dietary nutritional group } & \multirow{2}{*}{$\begin{array}{l}\text { Related studies } \\
\text { Abdul-Razzak } \\
\text { et al, [33], 2014 }\end{array}$} & \multirow{2}{*}{$\begin{array}{l}\text { Nutritional assessment } \\
\begin{array}{l}\text { Frequency and type of dairy product intake } \\
\text { (milk, yogurt, cheese, and labanah }\end{array}\end{array}$} & \multirow{2}{*}{$\begin{array}{l}\text { Relation with dysmenorrhea } \\
\text { There was significant reduction in } \\
\text { prevalence of very severe menstrual pain } \\
\text { by increasing daily dairy servings. }\end{array}$} \\
\hline & & & & \\
\hline & & $\begin{array}{l}\text { Karacin et al. [59], } \\
2018\end{array}$ & Consumption of dairy products & $\begin{array}{l}\text { The dysmenorrhea group had significantly } \\
\text { less consumption of dairy products }\end{array}$ \\
\hline \multirow[t]{17}{*}{$\begin{array}{l}\text { Other } \\
\text { products }\end{array}$} & \multirow[t]{7}{*}{ Sugar } & $\begin{array}{l}\text { Ozerdogan } \\
\text { et al. [29], } 2009\end{array}$ & $\begin{array}{l}\text { Sugar intake based on self-reported excessive } \\
\text { consumption of bread, table sugar, cola, } \\
\text { chocolate, rice, carrots, ice cream, cake, } \\
\text { yoghurt, noodles, milk, and sweets in daily diet }\end{array}$ & $\begin{array}{l}\text { Significantly higher risk of dysmenorrhea } \\
\text { in women consuming excessive sugar } \\
\text { compared with women with low intake }\end{array}$ \\
\hline & & $\begin{array}{l}\text { Tavallaee } \\
\text { et al. [16], } 2011\end{array}$ & Consumption of sweets and chocolate & $\begin{array}{l}\text { No association with menstrual } \\
\text { pain was found }\end{array}$ \\
\hline & & $\begin{array}{l}\text { Gagua } \\
\text { et al. [6], } 2012\end{array}$ & $\begin{array}{l}\text { Excessive sugar intake based on individual } \\
\text { self-report of excessive consumption of table } \\
\text { sugar, soda, chocolate, ice cream, and sweets in } \\
\text { their daily diet }\end{array}$ & $\begin{array}{l}\text { Increased dysmenorrhea was associated } \\
\text { with increased intake of sugar }\end{array}$ \\
\hline & & Grandi et al. [44], 2012 & Excessive sugar consumption & No association with dysmenorrhea \\
\hline & & $\begin{array}{l}\text { Mohamadirizi and } \\
\text { Kordi [52], } 2015\end{array}$ & $\begin{array}{l}\text { Consumption of sweet-fatty foods (chocolates, } \\
\text { cake, ice cream, nuts, etc.) }\end{array}$ & No association with dysmenorrhea \\
\hline & & Abu Helwa [55], 2018 & Sweets and sugary beverages & $\begin{array}{l}\text { No significant difference according to } \\
\text { dysmenorrhea }\end{array}$ \\
\hline & & $\begin{array}{l}\text { Muluneh et al. [60], } \\
2018\end{array}$ & Sugar intake & $\begin{array}{l}\text { Statistically increased risk of } \\
\text { dysmenorrhea was associated } \\
\text { with sugar intake }\end{array}$ \\
\hline & \multirow[t]{2}{*}{ Fat } & Nagata [39], 2005 & $\begin{array}{l}\text { Total fat, saturated fat, monounsaturated fat, } \\
\text { polyunsaturated fat }\end{array}$ & $\begin{array}{l}\text { Only saturated fat intake had positive } \\
\text { association with lower menstrual pain }\end{array}$ \\
\hline & & Tavallaee, [16] 2011 & Consumption of fat and oil & $\begin{array}{l}\text { Women who tended to have a low fat diet } \\
\text { significantly experienced less pain during } \\
\text { their menstrual periods }\end{array}$ \\
\hline & \multirow[t]{3}{*}{ Salt } & Ozerdogan [29], 2009 & Excessive salt intake & $\begin{array}{l}\text { Significantly higher prevalence of } \\
\text { dysmenorrhea in women who reported } \\
\text { consuming a moderate or excessive intake } \\
\text { of salt compared with women who had a } \\
\text { minimal intake }\end{array}$ \\
\hline & & Grandi et al. [44], 2012 & Excessive salt consumption & No association with dysmenorrhea \\
\hline & & Abu Helwa, [55], 2016 & Salty foods & $\begin{array}{l}\text { No significant difference according to } \\
\text { dysmenorrhea }\end{array}$ \\
\hline & \multirow[t]{5}{*}{ Caffeine } & Wang [37], 2004 & Tea consumption & $\begin{array}{l}\text { Significantly more tea consumption } \\
\text { among dysmenorrheic women }\end{array}$ \\
\hline & & Chung [38], 2005 & Coffee consumption & $\begin{array}{l}\text { No correlation between coffee } \\
\text { consumption and dysmenorrhea. }\end{array}$ \\
\hline & & Ozerdogan [29], 2009 & $\begin{array}{l}\text { Intake of caffeine including caffeinated soft } \\
\text { drinks, coffee, decaffeinated coffee, tea, } \\
\text { chocolate milk, and chocolate bars }\end{array}$ & $\begin{array}{l}\text { No correlation between caffeine } \\
\text { consumption and dysmenorrhea. }\end{array}$ \\
\hline & & Unsal et al. [28], 2010 & $\begin{array}{l}\text { Consumption of coffee, tea, cola, } \\
\text { and chocolate }\end{array}$ & $\begin{array}{l}\text { Significant correlation of dysmenorrhea } \\
\text { with coffee consumption but not tea, cola } \\
\text { and Chocolate consumption }\end{array}$ \\
\hline & & $\begin{array}{l}\text { Bin Mahmoud [43], } \\
2011\end{array}$ & $\begin{array}{l}\text { Daily consumption of the caffeinated } \\
\text { beverages including coffee, tea, Nescafe, } \\
\text { carbonated soft drinks, chocolate and hot } \\
\text { chocolate, in number of cups or cans per day }\end{array}$ & $\begin{array}{l}\text { Nescafe was a risk factor for menstrual } \\
\text { symptoms but No significant risk seen } \\
\text { with drinking tea or carbonated } \\
\text { soft drinks }\end{array}$ \\
\hline
\end{tabular}


Table 2 (continued)

\begin{tabular}{|c|c|c|c|}
\hline Dietary nutritional group & Related studies & Nutritional assessment & Relation with dysmenorrhea \\
\hline & Tavallaee et al. [16], 2011 & Coffee consumption & No association with dysmenorrhea \\
\hline & Grandi et al. [44], 2012 & Coffee consumption & No association with dysmenorrhea \\
\hline & Seven [45], 2013 & Coffee, tea and chocolate consumption & $\begin{array}{l}\text { Coffee and tea had no association with } \\
\text { dysmenorrhea, but chocolate eating } \\
\text { significantly affect dysmenorrhea }\end{array}$ \\
\hline & Sahin [48], 2014 & Cola, tea, coffee, chocolate consumption & No association with dysmenorrhea \\
\hline & Faramarzi [49], 2014 & $\begin{array}{l}\text { Caffeine intake including caffeinated } \\
\text { soft drinks, coffee, decaffeinated coffee, tea, } \\
\text { chocolate milk, and chocolate bars in } \\
\text { the daily diet }\end{array}$ & $\begin{array}{l}\text { High caffeine intake had significant } \\
\text { association with dysmenorrhea }\end{array}$ \\
\hline & $\begin{array}{l}\text { Mohamadirizi and } \\
\text { Kordi [52], } 2015\end{array}$ & $\begin{array}{l}\text { Consumption of caffeine-containing materials } \\
\text { (tea, coffee, etc.). }\end{array}$ & No association with dysmenorrhea \\
\hline & Abu Helwa [55], 2018 & Tea/coffee/Nescafe & No association with dysmenorrhea \\
\hline & $\begin{array}{l}\text { Pejčić and } \\
\text { Jankovic [57], } 2016\end{array}$ & Daily coffee intake & $\begin{array}{l}\text { Significantly associated with } \\
\text { dysmenorrhea }\end{array}$ \\
\hline & $\begin{array}{l}\text { Hailemeskel et al. [56], } \\
2016\end{array}$ & $\begin{array}{l}\text { Tea, Coca-Cola or Pepsi and Chocolate } \\
\text { consumption per day }\end{array}$ & $\begin{array}{l}\text { Significantly associated with } \\
\text { dysmenorrhea }\end{array}$ \\
\hline & $\begin{array}{l}\text { Muluneh } \\
\text { et al. [60], } 2018\end{array}$ & Daily coffee and tea intake & $\begin{array}{l}\text { Tea was not significantly associated but } \\
\text { coffee more than } 5 \text { cups per day was } \\
\text { significantly associated with } \\
\text { dysmenorrhea }\end{array}$ \\
\hline $\begin{array}{l}\text { Vitamins } \\
\text { and } \\
\text { minerals }\end{array}$ & Weissman et al. [17], 2004 & Vitamin use & $\begin{array}{l}\text { No significant association between } \\
\text { vitamin use and intensity of dysmenorrhe }\end{array}$ \\
\hline & Venkata [42], 2011 & Vitamin E \& C blood level & $\begin{array}{l}\text { Significantly decreased blood levels of } \\
\text { Vitamin E,C in cases with primary } \\
\text { dysmenorrhea }\end{array}$ \\
\hline & $\begin{array}{l}\text { Abdul-Razzak et al. [33], } \\
2014\end{array}$ & Plasma vitamin D concentration & $\begin{array}{l}\text { A high prevalence of vitamin D } \\
\text { insufficiency in participants with severe } \\
\text { and very severe dysmenorrhea. }\end{array}$ \\
\hline & Pramanik et al. [53], 2015 & $\begin{array}{l}\text { Daily intake of beta carotene, vitamin-E, } \\
\text { vitamin-C and zinc }\end{array}$ & $\begin{array}{l}\text { Significantly higher daily intake of beta } \\
\text { carotene, vitamin-E and zinc in girls } \\
\text { without dysmenorrhea than } \\
\text { dysmenorrheic counterpart. But no } \\
\text { difference according to vitamin C }\end{array}$ \\
\hline Fiber & Nagata et al. [39], 2005 & Dietary fiber & $\begin{array}{l}\text { Negative and significant association } \\
\text { between dietary fiber and intensity of } \\
\text { dysmenorrhea }\end{array}$ \\
\hline
\end{tabular}

their counterparts with dysmenorrhea. No difference was reported with vitamin C. Weissman et al. [17] reported a lack of significant relationship between vitamin intake and dysmenorrhea. Only Abdul-Razzak et al. [33] reported that individuals with severe and very severe dysmenorrhea had vitamin $\mathrm{D}$ deficiency or had low calcium intake. Nevertheless, there was no relationship between the severity of menstrual pain, and vitamin $\mathrm{D}$ and plasma calcium levels.

Fiber Consumption: A study examined the association between fiber consumption and dysmenorrhea, and reported a negative and significant relationship between them [39].

Nutrition as a Potential Factor of Primary Dysmenorrhea

\section{Results of the Studies According to Nutritional Habits}

Following Diet to Lose Weight: Montero et al. [35], Fujiwara [61], Rupa Vani et al. [46], and Hailemeskel et al. [56] studied the association between the current or previous attempts of going on a diet to lose weight and the intensity of dysmenorrhea. Consistently, a positive association between following a diet to lose weight and dysmenorrhea was reported.

Following Vegetarian Diet: Only one study focused on the relationship between having a vegetarian diet and dysmenorrhea. No significant difference was found in the prevalence of dysmenorrhea in vegetarian and non-vegetarian subjects [47]. 
Table 3. Summarized results of reviewed studies according to nutritional habits

\begin{tabular}{|c|c|c|c|}
\hline $\begin{array}{l}\text { Nutritional } \\
\text { habit }\end{array}$ & Related studies & Nutritional assessment & Relation with dysmenorrhea \\
\hline \multirow[t]{2}{*}{$\begin{array}{l}\text { Following diet } \\
\text { to lose weight }\end{array}$} & $\begin{array}{l}\text { Montero } \\
\text { et al. [35], } 1996\end{array}$ & Dieting for loose weight & $\begin{array}{l}\text { Attempting to lose weight was significantly } \\
\text { associated with increased menstrual pain }\end{array}$ \\
\hline & Fujiwara [61], 2007 & $\begin{array}{l}\text { Having no experience with dieting/ } \\
\text { currently on a diet/having history of dieting }\end{array}$ & $\begin{array}{l}\text { Participants with history of dieting had higher } \\
\text { intensity of dysmenorrhea than the other groups }\end{array}$ \\
\hline $\begin{array}{l}\text { Following } \\
\text { vegetarian diet }\end{array}$ & Gangwar et al. [47], 2014 & $\begin{array}{l}\text { Dietary habits as vegetarian/ } \\
\text { non-vegetarian }\end{array}$ & $\begin{array}{l}\text { No significant difference in the prevalence of } \\
\text { dysmenorrhea in vegetarian and } \\
\text { non-vegetarian subjects }\end{array}$ \\
\hline \multirow[t]{13}{*}{$\begin{array}{l}\text { Eating } \\
\text { behavior }\end{array}$} & Fujiwara [36], 2003 & Skipping breakfast & $\begin{array}{l}\text { A significant correlation between skipping } \\
\text { breakfast and intensity of dysmenorrhea }\end{array}$ \\
\hline & $\begin{array}{l}\text { Fujiwara and } \\
\text { Nakata [41], } 2010\end{array}$ & $\begin{array}{l}\text { Having breakfast every morning/having } \\
\text { breakfast } 1-6 \text { times a week/having } \\
\text { breakfast less than once a week }\end{array}$ & $\begin{array}{l}\text { Severity of dysmenorrhea was significantly } \\
\text { higher in the population that skipped breakfast }\end{array}$ \\
\hline & Gagua et al. [6], 2012 & $\begin{array}{l}\text { Meal skipping based on number of meal } \\
\text { intakes throughout the } 24 \mathrm{~h}\end{array}$ & $\begin{array}{l}\text { Meal skipping (inadequate nutrition) was } \\
\text { one of most important risk factors } \\
\text { of dysmenorrhea }\end{array}$ \\
\hline & Grandi et al. [44], 2012 & Attitude to eating breakfast & No association with dysmenorrhea \\
\hline & $\begin{array}{l}\text { Fujiwara and } \\
\text { Nakata [32], } 2013\end{array}$ & Skipping breakfast & $\begin{array}{l}\text { Significantly higher severity of dysmenorrhea } \\
\text { in women that skipped breakfast }\end{array}$ \\
\hline & Rupa Vani et al. [46], 2013 & Eating junk foods & No association with dysmenorrhea \\
\hline & $\begin{array}{l}\text { Faramarzi and } \\
\text { Salmalian [49], } 2014\end{array}$ & Having breakfast & No significant relation with dysmenorrhea \\
\hline & Jeon et al. [50], 2014 & Regular/Irregular eating & $\begin{array}{l}\text { The score for dysmenorrhea was significantly } \\
\text { higher when the participants ate meals irregularly }\end{array}$ \\
\hline & Kazama et al. [51], 2015 & Having breakfast & $\begin{array}{l}\text { Likelihood of experiencing dysmenorrhea } \\
\text { significantly increased when sometimes } \\
\text { eating breakfast or skipped breakfast }\end{array}$ \\
\hline & $\begin{array}{l}\text { Mohamadirizi and } \\
\text { Kordi [52], } 2015\end{array}$ & $\begin{array}{l}\text { Having fast foods like hamburger, } \\
\text { pizza, potato chips, etc. }\end{array}$ & No significant relation with dysmenorrhea \\
\hline & Bavil et al. [54], 2016 & Eating behavior & $\begin{array}{l}\text { Significant differences between the groups with } \\
\text { and without dysmenorrhea in terms of } \\
\text { eating behavior }\end{array}$ \\
\hline & Abu Helwa [55], 2018 & Skipping breakfast & $\begin{array}{l}\text { Skipping breakfast was the only significant } \\
\text { predictors of moderate/severe dysmenorrhea }\end{array}$ \\
\hline & $\begin{array}{l}\text { Shinde and } \\
\text { Laddad [58], } 2016\end{array}$ & Eating junk foods & $\begin{array}{l}\text { Dysmenorrhea were more frequent in girls with } \\
\text { habit of eating junk food }<3 \text { days a week }\end{array}$ \\
\hline
\end{tabular}

Eating Behavior: Eating behaviors such as skipping breakfast, irregular eating, fast foods, or process foods were studied. Skipping breakfast was the most studied eating behavior and 6 studies found a significant correlation between skipping breakfast and the intensity of dysmenorrhea. Those women who skipped breakfast suf- fered from a higher intensity of dysmenorrhea [32, 36, 40, 41, 51, 55]. Conversely, Faramarzi and Salmalian [49] did not find a significant relationship between eating breakfast and dysmenorrhea. Also, no association was observed between the attitude toward eating breakfast and dysmenorrhea [44]. 
Habits of regular eating or meal skipping were studied by Gagua et al. [6] and Jeon et al. [50]. They showed that those subjects with irregular and skipped meal habits had a significantly higher intensity of dysmenorrhea. Also Bavil et al. [54] found significant differences between the groups with and without dysmenorrhea in terms of eating behaviors.

Studies on eating fast foods/process foods/junk foods reported inconsistent results. Fujiwara et al. [40] and Shinde and Laddad [58] reported a significantly higher level of dysmenorrhea in those subjects who ate fast foods and processed foods compared to their healthy counterparts. Mohamadirizi and Kordi [54] and Rupa Vani et al. [46] did not report such an association.

\section{Discussion}

Dysmenorrhea is one of the main factors influencing the quality of life and social activities of women [1,8]. Several factors can affect primary dysmenorrhea and its incidence and severity. Nutrition has not been adequately studied with regard to its effect on dysmenorrhea [21, 27]. Therefore, this systematic review aimed to investigate the association between primary dysmenorrhea and dietary habits.

Regarding dietary habits and the consumption of different nutritional groups, the consumption of carbohydrates including bread, cereals, meat, legumes, and nuts had no association with dysmenorrhea and its intensity. Consumption of fish and eggs as the alternatives of meat showed inconsistent results. Regarding the association between fish and dysmenorrhea, the consumption of fish was associated with less dysmenorrhea pain, but 2 studies found inconsistent results. This finding is consistent with results of recent studies indicating the effects of fish oil on primary dysmenorrhea $[62,63]$. The prevention of prostaglandins synthesis is the major mechanism of fish oil [20]. The protective role of fish is due to the content of omega- 3 fatty acids. If more dietary omega- 3 fatty acids are consumed, they enter the phospholipid membrane [64]. During menstruation, omega-3 fatty acids compete with fatty acids containing omega- 6 to produce prostaglandins and leukotriene. In addition, they can hinder the production of arachidonic acid [27]. The effect of arachidonic acid in animal fats, especially meat, is the synthesis of prostaglandin [54]. Prostaglandins produced from omega-3 fatty acids are less potent, and can reduce the contraction of the myometrium and vessels [16]. A reduction of systemic symptoms is due to a poor leukotriene's production. Egg can prevent dysmenorrhea due to its magnesium and calcium contents. Magnesium such as calcium plays a key role in the membrane fixation mechanism and can modulate synapses through calcium-de-

Nutrition as a Potential Factor of Primary Dysmenorrhea pendent mechanisms. Reductions in magnesium levels increase synaptic transmission, resulting in myometric muscle contractions and, consequently, dysmenorrhea [27]. Foods containing the high levels of magnesium can reduce the severity of dysmenorrhea through reducing the synthesis of prostaglandins and spasticity of small muscles and vessels [54]. However, such findings are not consistent with that of the current systematic review. Such inconsistency may be due to small numbers of studies in this subgroup.

Studies on the association between the consumption of fruits and vegetables and dysmenorrhea were inconsistent. Two of 4 studies in this subgroup showed no significant association [26,55], but 2 other studies confirmed that the increased consumption of fruits and vegetables are associated with the reduction of menstrual pain [16, 27]. Fruits and vegetables are important sources of vitamins and antioxidants [65]. Therefore, the combination of these results with the findings of studies on the plasma level of vitamins confirmed that women with dysmenorrhea took less fruits and vegetables and had less plasma levels of vitamins [33, 42, 53]. Vitamins, beta-carotene, zinc, and minerals are diet antioxidants. Vitamin $\mathrm{E}$ is fat soluble and is primarily responsible for oxidant membrane injuries and is the first line of defense against the peroxidation of phospholipids. Vitamin C (ascorbic acid) is soluble in water and provides an antioxidant capacity by removing oxygen-free radicals. So vitamin $\mathrm{C}$ can convert vitamin-E-free radical back to vitamin $\mathrm{E}$ and are essential for the recycling of vitamin $\mathrm{E}$ to prevent fat peroxidation. Beta carotene inhibits oxidants. Zinc is an antioxidant factor [53]. Reducing the level of antioxidants can increase the consumption of antioxidants or free radicals to detoxify the increased levels of oxidants in primary dysmenorrhea. A study on vitamins E and B (thiamine), magnesium, and fish oil suggests that these foods are important sources of vitamins E, A, and other nutrients [7]. Women with primary dysmenorrhea who use non-steroidal anti-inflammatory drugs are suggested to use natural antioxidants such as vitamin $\mathrm{E}$, vitamin $\mathrm{C}$, and $\beta$-carotenoids for the reduction of pain $[7,53]$. Single randomized controlled trials showed the effect of vitamin supplements on the reduction of menstrual pain reduction $[63,66,67]$, but a Cochrane meta-analysis on the effect of dietary supplements for primary dysmenorrhea lacked high quality evidence to support the effectiveness and safety of any dietary supplement for dysmenorrhea. Therefore, more research with higher methodological qualities are required [24].

Five studies assessed the association between the dairy consumption (calcium intake) and dysmenorrhea and 
they mostly (4 out of 5) reported a positive association between dairy consumption and the reduction of menstrual pain $[16,21,33,59]$. Calcium intake in the diet has a protective effect on dysmenorrhea [21]. Calcium is absorbed in the upper intestine and can regulate the ability of muscle cells to respond to nerve stimuli through various functions, which is called stabilizing [21, 27, 33]. Therefore, dairy and calcium consumption can reduce dysmenorrhea through controlling muscle nervous activities. Conversely, reduction of calcium concentrations can increase muscle spasm, spasticity, and muscle contractions [21]. The possible explanation for this relationship is that the absorption and metabolism of vitamins and minerals are endangered by the use of sugar leading to muscle spasm and menstrual pain [56].

Sugar consumption had an inconsistent association with dysmenorrhea. Excessive sugar intake was increased by the absorption of certain vitamins and minerals and led to a food instability. It creates muscle spasm and pain in the menstrual cycle. Also, certain nutrients directly influence the circulation status of sexual steroids and cause muscle spasm $[6,29,48,56]$. The relationship between caffeine intake and dysmenorrhea has been reported in many studies due to its effect on vasoconstriction and pelvic pain [49]. On the other hand, caffeine can stimulate stress and irritability $[28,37,49,56]$.

Nutritional deficiencies are the most important factors that disrupt the hypothalamic-pituitary-ovarian axis. Nutritional deficiencies such as not eating breakfast contribute to the development of gynecological disorders especially endometriosis [36]. The nutritional status influences sexual steroids. Insufficient nutrition causes a change in hormonal levels due to reduced levels of energy [6]. Menstrual pain occurs only during ovulation. Progesterone affects the synthesis of prostaglandins and their attachment to myometrium receptors. Prostaglandins affect the uterine muscle and vascular tone, and dysmenorrhea is caused by an imbalance in prostaglandins. In addition, progesterone has some effects on the concentration of prostaglandin in myometrium [37].

The majority of the studies had issues in reporting potential source of bias, sample size estimation, description of design, and statistical analysis of quantitative variables. So there is a need to design and implement studies with stronger methodologies.

\section{Limitations}

The most important limitation of this review study was methodological heterogeneity of studies. The heterogeneity in the assessment and report of consumption of nu- tritional groups and habits limited the data synthesis to qualitative synthesis. Performing meta-analysis was impossible. Qualitative synthesis has limitations in terms of providing conclusive findings. A lack of pre-registration protocol and exclusion of studies publications prior to 1990 and published in other languages are the other limitations of this study.

\section{Conclusion}

This systematic review showed the relationship between some nutritional factors and primary dysmenorrhea, indicating the need for further studies on this topic. The increased consumption of fruits and vegetables as the sources of many vitamins and minerals, fish and milk, and dairy can have a positive association with less menstrual pain. Other findings are related to nutritional habits, but most studies showed a negative association between dysmenorrhea and meal skipping and following diet to lose weight. Therefore, attention should be paid to the correction of female nutritional behaviors for having enough and balanced diet. Given the negative effects of dysmenorrhea on the quality of life, measures should be taken to increase the knowledge of women about dysmenorrhea and make appropriate lifestyle changes to control it. Designing and implementing interventional studies on nutrition education and modification are recommended.

\section{Disclosure Statement}

The authors declare no conflicts of interest.

\section{Financial Support}

None.

References

1 Iacovides S, Avidon I, Baker FC: What we know about primary dysmenorrhea today: a critical review. Hum Reprod Update 2015;21: 762-778.

2 Jonatan S, Berek J: Novak's Gynecology: 14th. New York, Lippincott Williams and Wilkins Publishers, 2012.

3 Chuamoor K, Kaewmanee K, Tanmahasamut P: Dysmenorrhea among Siriraj nurses; prevalence, quality of life, and knowledge of management. J Med Assoc Thai 2012;95: 983-991. 
4 Mathias SD, Kuppermann M, Liberman RF, Lipschutz RC, Steege JF: Chronic pelvic pain: prevalence, health-related quality of life, and economic correlates. Obstet Gynecol 1996;87: 321-327.

5 Shewte MK, Sirpurkar MS: Dysmenorrhoea and quality of life among medical and nursing students: a cross-sectional study. Nat J Comm Med 2016;7:474-479.

6 Gagua T, Tkeshelashvili B, Gagua D: Primary dysmenorrhea: prevalence in adolescent population of Tbilisi, Georgia and risk factors. J Turk Ger Gynecol Assoc 2012;13:162-168.

7 Haidari F, Akrami A, Sarhadi M, Mohammad Shahi M: Prevalence and severity of primary dysmenorrhea and its relation to anthropometric parameters. Tums Hayat 2011;17:7077.

$8 \mathrm{Ju} \mathrm{H}$, Jones M, Mishra G: The prevalence and risk factors of dysmenorrhea. Epidemiol Rev 2014;36:104-113.

9 Burnett MA, Antao V, Black A, Feldman K, Grenville A, Lea R, et al: Prevalence of primary dysmenorrhea in Canada J Obstet Gynaecol Can 2005;27:765-770.

10 Latthe P, Mignini L, Gray R, Hills R, Khan K: Factors predisposing women to chronic pelvic pain: systematic review. BMJ 2006;332: 749-755.

11 Nohara M, Momoeda M, Kubota T, Nakabayashi M. Menstrual cycle and menstrual pain problems and related risk factors among Japanese female workers. Ind Health 2011;49: 228-234.

12 Ohde S, Tokuda Y, Takahashi O, Yanai H, Hinohara S, Fukui T: Dysmenorrhea among Japanese women. Int J Gynaecol Obstet 2008; 100:13-17.

13 Patel V, Tanksale V, Sahasrabhojanee M Gupte S, Nevrekar P: The burden and determinants of dysmenorrhoea: a populationbased survey of 2262 women in Goa, India. BJOG 2006; 113:453-463.

14 Pawłowski B: Prevalence of menstrual pain in relation to the reproductive life history of women from the Mayan rural community. Ann Hum Biol 2004;31:1-8.

15 Santer M, Warner P, Wyke S: A scottish postal survey suggested that the prevailing clinical preoccupation with heavy periods does not reflect the epidemiology of reported symptoms and problems. J Clin Epidemiol 2005;58: 1206-1210.

16 Tavallaee M, Joffres MR, Corber SJ, Bayanzadeh M, Rad MM: The prevalence of menstrual pain and associated risk factors among Iranian women. J Obstet Gynaecol Res 2011;37: 442-451.

17 Weissman AM, Hartz AJ, Hansen MD, Johnson SR: The natural history of primary dysmenorrhoea: a longitudinal study. BJOG 2004;111:345-352.

18 Osayande AS, Mehulic S: Diagnosis and initial management of dysmenorrhea. Am Fam Physician 2014;89:341-346.

19 Marjoribanks J, Proctor M, Farquhar C Derks RS: Nonsteroidal anti-inflammatory drugs for dysmenorrhoea. Cochrane Database Syst Rev 2015;7:CD001751.

20 Proctor M, Farquhar C: Diagnosis and management of dysmenorrhoea. BMJ 2006;332: 1134-1138.

21 Abdul-Razzak KK, Ayoub NM, Abu-Taleb AA, Obeidat BA: Influence of dietary intake of dairy products on dysmenorrhea. J Obstet Gynaecol Res. 2010;36:377-383.

22 Proctor ML, Murphy PA: Herbal and dietary therapies for primary and secondary dysmenorrhoea. Cochrane Database Syst Rev 2001;3: CD002124.

23 Sidani M, Campbell J: Gynecology: select topics. Prim Care 2002;29:297-321.

24 Pattanittum P, Kunyanone N, Brown J, Sangkomkamhang US, Barnes J, Seyfoddin V, et al: Dietary supplements for dysmenorrhoea. Cochrane Database Syst Rev 2016;3: CD002124.

25 Zhu X, Proctor M, Bensoussan A, Wu E, Smith CA: Chinese herbal medicine for primary dysmenorrhoea. Cochrane Database Syst Rev 2008;2:CD005288.

26 Di Cintio E, Parazzini F, Tozzi L, Luchini L Mezzopane R, Marchini M, et al: Dietary habits, reproductive and menstrual factors and risk of dysmenorrhoea. Eur J Epidemiol 1997; 13:925-930.

27 Balbi C, Musone R, Menditto A, Di Prisco L, Cassese E, D'Ajello M, et al: Influence of menstrual factors and dietary habits on menstrual pain in adolescence age. Eur J Obstet Gynecol Reprod Biol 2000;91:143-148.

28 Unsal A, Ayranci U, Tozun M, Arslan G, Calik E: Prevalence of dysmenorrhea and its effect on quality of life among a group of female university students. Ups J Med Sci 2010; 115:138-145

29 Ozerdogan N, Sayiner D, Ayranci U, Unsal A, Giray S: Prevalence and predictors of dysmenorrhea among students at a university in Turkey. Int J Gynaecol Obstet 2009;107:3943.

30 Moher D, Liberati A, Tetzlaff J, Altman DG; PRISMA Group: Preferred reporting items for systematic reviews and meta-analyses: the PRISMA statement. PLoS Med.2009;6: e1000097.

31 von Elm E, Altman DG, Egger M, Pocock SJ, Gøtzsche PC, Vandenbroucke JP: The strengthening the reporting of observational studies in epidemiology (STROBE) statement: guidelines for reporting observational studies. PLoS Med 2007;4:e296.

32 Fujiwara T, Nakata R: Skipping breakfast may induce dysmenorrhea and irregular menstruation in post-adolescent women in Japan. Ann Nutr Metab 2013;63:668.

33 Abdul-Razzak KK, Obeidat BA, Al-Farras MI, Dauod AS: Vitamin D and PTH status among adolescent and young females with severe dysmenorrhea. J Pediatr Adolesc Gynecol. 2014;27:78-82.

34 Deutch B: Menstrual pain in Danish women correlated with low $n-3$ polyunsaturated fatty acid intake. Eur J Clin Nutr 1995;49:508-516.
35 Montero P, Bernis C, Fernandez V, Castro S: Influence of body mass index and slimming habits on menstrual pain and cycle irregularity. J biosoc Sci. 1996;28:315-323.

36 Fujiwara T: Skipping breakfast is associated with dysmenorrhea in young women in Japan. Int J Food Sci Nutr 2003;54:505509.

37 Wang L, Wang X, Wang W, Chen C, Ronnennberg AG, Guang W, et al: Stress and dysmenorrhoea: a population based prospective study. Occup Environ Med 2004;61:10211026.

38 Chung FF, Yao CC, Wan GH: The associations between menstrual function and life style/working conditions among nurses in Taiwan. J Occup Health 2005;47:149156.

39 Nagata C, Hirokawa K, Shimizu N, Shimizu $\mathrm{H}$ : Associations of menstrual pain with intakes of soy, fat and dietary fiber in Japanese women. Eur j clin nutr 2005;59:88-92.

40 Fujiwara T, Sato N, Awaji H, Sakamoto $\mathrm{H}$, Nakata R: Skipping breakfast adversely affects menstrual disorders in young college students. Int J Food Sci Nutr 2009;60:23-31.

41 Fujiwara T, Nakata R: Skipping breakfast is associated with reproductive dysfunction in post-adolescent female college students. Appetite 2010;55:714-717.

42 Venkata RS, Kiran VR, Vijayasree M: Oxidative stress and antioxidant status in primary dysmenorrhea. J Clin Diagn Res 2011 5:509511

43 Bin Mahmoud AZ, Makhdoom AN, Mufti LA, Alreheli RS, Farghal RG, Aljaouni SE: Association between menstrual disturbances and habitual use of caffeine. J Taibah University Med Sci 2014;9:341-344.

44 Grandi G, Ferrari S, Xholli A, Cannoletta M, Palma F, Romani C, et al: Prevalence of menstrual pain in young women: what is dysmenorrhea? J Pain Res. 2012;5:169-174.

45 Seven M, Guvenc G, Akyuz A, Eski F: Evaluating dysmenorrhea in a sample of Turkish nursing students. Pain Manag Nurs 2013;15: 664-671.

46 Vani R, Veena S, Subitha L, Hemanth Kumar $\mathrm{R}$, Bupathy A: Menstrual abnormalities in school going girls - are they related to dietary and exercise pattern? J Clin Diagn Res 2013;7: 2537-2540.

47 Gangwar V, Kumar D, Gangwar RS, Arya M, Banoo H: Prevalence of primary dysmenorrhea among the undergraduate medical students and its impact on their performance in study. Int J Physiol 2014;2:14.

48 Sahin S, Ozdemir K, Unsal A, Arslan R: Review of frequency of dysmenorrhea and some associated factors and evaluation of the relationship between dysmenorrhea and sleep quality in university students. Gynecol Obstet Invest 2014;78:179-185.

49 Faramarzi M, Salmalian H: Association of psychologic and nonpsychologic factors with primary dysmenorrhea. Iran Red Crescent Med J 2014;16:e16307. 
50 Jeon GE, Cha NH, Sok SR: Factors influencing the dysmenorrhea among korean adolescents in middle school. J Phys Ther Sci 2014; 26:1337-1343.

51 Kazama M, Maruyama K, Nakamura K: Prevalence of dysmenorrhea and its correlating lifestyle factors in Japanese female junior high school students. Tohoku J Exp Med 2015;236: 107-113.

52 Mohamadirizi S, Kordi M: The relationship between food frequency and menstrual distress in high school females. Iran J Nurs Midwifery Res 2015;20:689-693.

53 Pramanik P, Banerjee SB, Saha P: Primary dysmenorrhea in school going adolescent girls - is it related to deficiency of antioxidant in diet? Int J Life Sci Pharma Res 2015;5:L54L63.

54 Bavil DA, Dolatian M, Mahmoodi Z, Baghban AA: Comparison of lifestyles of young women with and without primary dysmenorrhea. Electron Physician 2016;8:2107-2114.

55 Abu Helwa HA, Mitaeb AA, Al-Hamshri S, Sweileh WM: Prevalence of dysmenorrhea and predictors of its pain intensity among Palestinian female university students. BMC Womens Health 2018;18:18.

56 Hailemeskel S, Demissie A, Assefa N: Primary dysmenorrhea magnitude, associated risk factors, and its effect on academic performance: evidence from female university students in
Ethiopia. Int J Womens Health 2016;8:489496.

57 Pejčić A, Jankovic S: Risk factors for dysmenorrhea among young adult female university students. Ann Ist Super Sanita 2016;52:98103.

58 Shinde GR, Laddad M: Overview of adolescent menstrual problems and its relation to bmi, eating habits and physical activity. J Evolution Med Dental Sci 2016;5:6757-6761.

59 Karacin O, Mutlu I, Kose M, Celik F, KanatPektas M, Yilmazer M: Serum vitamin D concentrations in young Turkish women with primary dysmenorrhea: a randomized controlled study. Taiwan J Obstet Gynecol 2018; 57:58-63.

60 Muluneh AA, Nigussie TS, Gebreslasie KZ, Anteneh KT, Kassa ZY: Prevalence and associated factors of dysmenorrhea among secondary and preparatory school students in Debremarkos town, North-West Ethiopia. BMC Womens Health 2018;18:57.

61 Fujiwara T: Diet during adolescence is a trigger for subsequent development of dysmenorrhea in young women. Int J Food Sci Nutr 2007;58:437-444.

62 Mehrpooya M, Eshraghi A, Rabiee S, LarkiHarchegani A, Ataei S: Comparison the effect of fish-oil and calcium supplementation on treatment of primary dysmenorrhea. Rev Recent Clin Trials 2017;12:148-153.
63 Sadeghi N, Paknezhad F, Rashidi Nooshabadi M, Kavianpour M, Jafari Rad S, Khadem Haghighian H: Vitamin E and fish oil, separately or in combination, on treatment of primary dysmenorrhea: a double-blind, randomized clinical trial. Gynecol Endocrinol 2018: 34:804-808.

64 Zafari M, Tofighi M, Aghamohammady A, Behmanesh F, Rakhshaee Z: Comparison of the effect of acupressure, fish oil capsules and ibuprofen on treatment of primary dysmenorrhea. Afr J Pharm Pharmacol 2011;5:11151119.

65 Saini RK, Nile SH, Park SW: Carotenoids from fruits and vegetables: chemistry, analysis, occurrence, bioavailability and biological activities. Food Res Int. 2015;76:735750

66 Moini A, Ebrahimi T, Shirzad N, Hosseini R, Radfar M, Bandarian F, et al: The effect of vitamin $\mathrm{D}$ on primary dysmenorrhea with vitamin D deficiency: a randomized double-blind controlled clinical trial. Gynecol Endocrinol 2016;32:502-505.

67 Zarei S, Mohammad-Alizadeh-Charandabi S, Mirghafourvand M, Javadzadeh Y, EffatiDaryani F: Effects of calcium-vitamin D and calcium-alone on pain intensity and menstrual blood loss in women with primary dysmenorrhea: a randomized controlled trial. Pain Med 2017;18:3-13. 